\title{
Incidencia de la Convertibilidad sobre los Sectores Productivos y el Empleo en la Provincia de Corrientes*
}

\author{
Lucas Gómez Tonsich **
}

\section{Resumen}

El presente trabajo tiene por finalidad dar cuenta de las trasformaciones operadas en la economía de la provincia de Corrientes a partir de los acontecimientos ocurridos a nivel nacional, con especial detalle en los problemas generados en los mercados laborales tanto nacional como provincial.

A partir de una breve descripción de las tendencias recientes en la economía mundial y de un repaso de los puntos del llamado "Consenso de Washington", se expondrán las circunstancias en que se implementó el régimen de convertibilidad en la Argentina y se detallarán las repercusiones que tuvo sobre la producción nacional. Luego se caracterizarán la economía de la provincia de Corrientes, los rasgos principales de su inserción productiva y los cambios que en ella operaron durante la vigencia del régimen de convertibilidad. En una segunda instancia se examinará la evolución del mercado laboral nacional y provincial a partir de los principales indicadores para señalar los efectos del fenómeno de "trabajador adicional" tuvo en la dinámica del mercado, así como explorar sus posibles causas. Finalmente se analizará la dinámica de empleo de los distintos sectores productivos durante la etapa final del régimen de convertibilidad a partir de la información sobre asalariados registrados del sector privado.

\footnotetext{
* Artículo recibido 9 de abril de 2012. Aceptado 11 de noviembre de 2012.

** Contador Público (UNNE). Maestrando en Ciencias Sociales (UNQ). Auxiliar Docente. Cátedra de Historia Económica. Facultad de Ciencias Económicas (UNNE). Becario de Posgrado (CONICET), Argentina.
} 
El enfoque metodológico adoptado es cuantitativo y el nivel de investigación es descriptivo transversal, comprendiendo el análisis sectorial del producto, el empleo y las remuneraciones. Las fuentes de datos son secundarias y corresponden a información relevada tanto por organismos nacionales (INDEC, ProvInfo, MTySS) como provinciales (DEyC).

\section{Palabras clave}

Convertibilidad - producción - empleo - Corrientes.

\section{Abstract}

This paper is intended to account for the transformations operated on the economy of the province of Corrientes from the events at the national level, with special emphasis on the problems arising in labor markets both national and provincial levels.

After a brief description of recent trends in the global economy and a review of the points of the "Washington Consensus", will describe the circumstances under which the currency board was implemented in Argentina and will detail the impact that had on the domestic industry.

Then characterized the economy of the province of Corrientes, the main features of their productive insertion, and the changes operated during the convertibility regime.

In a second instance will examine the evolution of national and provincial labor market from the main indicators to point out the effects of the phenomenon of "additional worker" had on the market dynamics and to explore its possible causes.

Finally, we analyze the dynamics of employment of productive sectors during the final stage of the convertibility regime from the information on registered private-sector employees.

The methodological approach is quantitative and the level of research is descriptive transversal, comprising sectorial analysis of output, employment and wages. Data sources are secondary and correspond to information collected by national -INDEC, ProvInfo, MTySS- and provincial -DEyC- agencies.

\section{Keywords}

Convertibility - Production - Employment - Corrientes. 


\section{La financiarización de la economía mundial a fines del siglo XX}

A comienzos de la década de 1970 se asistió al surgimiento de un nuevo orden financiero internacional como resultado de la conjunción de, en primer lugar, la crisis en el sistema de patrón oro-dólar construido a partir de los acuerdos de Bretton Woodsde 1944; por otro lado, el incremento de los déficits fiscales en los países desarrollados, que necesitados de recursos aumentaron artificialmente la liquidez mundial; en tercer lugar, el incremento en la cuantía de los denominados petrodólares que multiplicó y tornó más inestables los flujos de capital a nivel mundial y finalmente un cambio de estrategia de las compañías multinacionales que priorizaron sus actividades financieras en detrimento de su operatoria tradicional (Barbero, Saborido, Beremblum, López Nadal, \& Ojeda, 2007).

El establecimiento de nuevas pautas de organización y funcionamiento para las empresas, como consecuencia del empleo de las nuevas tecnologías, permitió que el comercio, la producción y los flujos de capitales tendieran a acentuar la internacionalización de la economía. Más que comercio libre, se asistía en esos años a un comercio mundial "administrado" debido al predominio de grandes corporaciones dentro de las cuales se desarrolló una parte importante del flujo internacional de productos. En las décadas de 1980 y 1990 alrededor del 40 \% del intercambio mundial de bienes lo constituyó el intercambio entre filiales de empresas multinacionales (Ferrer, 2006).

La transnacionalización de las empresas se acentuó en la segunda mitad del siglo XX con la expansión de la inversión extranjera directa y las fusiones de compañías, expresando una creciente asociación y concentración de capitales y empresas de diferentes naciones. Pero la mayor parte de las grandes firmas multinacionales continuó teniendo su sede y sus activos principales en los Estados Unidos, Alemania, Francia, Reino Unido y Japón, lo cual explica no solo el dominio económico de reducido número de empresas, sino la vigencia del rol hegemónico de algunas naciones y de las crecientes desigualdades del poder de decisión en el escenario económico y político mundial (Krugman \& Obstfeld, 2002).

Este nuevo régimen monetario mucho menos regulado coincidió, a partir de finales de la década de 1980, con una reducción de las tasas mundiales de inflación a raíz de las políticas monetarias ortodoxas aplicadas en las principales economías desarrolladas (v. g. Estados Unidos y Gran Bretaña) y la caída en los precios de los commodities (liderados por el petróleo) que redundaron en una disminución de las tasas de interés y consecuentemente una reversión de los flujos internacionales de capitales posaron nuevamente su mirada en las economías en desarrollo (Rapoport, 2005). 


\section{La opción neoliberal de Argentina}

Simultáneamente en nuestro país comenzaban a cobrar repercusión aquellas voces que, aun reconociendo que la sustitución de importaciones merecía crédito por tres décadas de crecimiento económico, señalaban la existencia de claros signos de pérdida de dinamismo y evidencia de desequilibrios macroeconómicos cada vez mayores.

Mencionaban la consolidación de sectores oligopólicos bajo el amparo de mercados domésticos protegidos, poco inclinados a la innovación tecnológica y de baja competitividad internacional; o la persistencia de patrones de integración económica internacional caracterizados por una fuerte vulnerabilidad dada su dependencia de un reducido número de productos sujetos a los vaivenes de los mercados mundiales; o la insatisfactoria creación de puestos de trabajo como resultado de la elevada intensidad de capital requerida por las nuevas inversiones.

Paradójicamente el colapso del orden económico internacional de posguerra a comienzos de la década de 1970 tuvo en la Argentina, y en América Latina en su conjunto, efectos opuestos a los del mundo desarrollado. Así, mientras estos últimos enfrentaron el nuevo escenario abandonando el paradigma industrial fordista y adoptando esquemas productivos más flexibles y orientados a intermediación financiera, los países de la región disfrutaron de una abundancia de capitales que les permitió ignorar las debilidades estructurales que aquejaban a sus economías y de esa manera posponer el ajuste (Thorp, 1998). Pero a partir de la década de 1980, merced a la crisis de la deuda externa, y más intensamente en la década siguiente en el marco del Ilamado "Consenso de Washington", se implementó en el país y en la región una serie de políticas de reforma e integración comercial cuyos objetivos fueron abandonar el modelo de sustitución de importaciones y promover un patrón de crecimiento orientado hacia el exterior.

El conjunto de recomendaciones para sanear las economías latinoamericanas fue conocido como el "Consenso de Washington" y tenía como ejes el control del gasto público y la disciplina fiscal en tanto eran identificados como fuentes de la excesiva emisión monetaria, la liberalización del comercio y del sistema financiero para tornar más competitivas a las economías, el fomento a la inversión extranjera como forma de suplir la carencia de ahorro interno, la privatización de las empresas públicas con miras a reducir el déficit fiscal y elevar la competitividad de las empresas vía reducción de las tarifas, y la desregulación y reforma del Estado para liberar las fuerzas productivas de la economía. 


\section{El régimen de convertibilidad}

La implementación del Régimen de Convertibilidad en la Argentina se dio en medio de los sucesivos brotes inflacionarios que caracterizaron el final de mandato de Raúl Alfonsín. La defensa de la moneda local (austral) por parte de las autoridades monetarias contra los ataques especulativos había dejado al Banco Central prácticamente sin reservas internacionales, por lo que a comienzos de 1989 el gobierno decidió reorganizar el mercado cambiario estableciendo dos mercados con precios regulados (tanto para exportaciones como para importaciones) y un tercero libre (para operaciones financieras) precipitando una masiva fuga de capitales (Ossona, 2001).

La cotización del dólar libre se disparó realimentando la corrida cambiaria al tiempo que los precios internos comenzaron a imitar el comportamiento de la divisa estadounidense, por lo que se asistió a un proceso hiperinflacionario. Entre las causas de dicha hiperinflación pueden mencionarse la crítica situación de la balanza de pagos a raíz de los cuantiosos pagos externos, la exacerbación de la puja distributiva al reinstaurarse las negociaciones colectivas, la especulación de importantes actores económicos tanto locales como foráneos, el acortamiento de los sucesivos ciclos de estabilización y crisis y finalmente la errática política del gobierno radical (Vitelli, 1999).

En medio de la agitación social que acompañó la asunción de Carlos Menen, y luego de unos primeros intentos de reforma menores, a comienzos de 1991 se anunció un nuevo plan económico a cargo del flamante ministro de economía Domingo Cavallo. El mencionado programa se sustentaba, en sintonía con las recomendaciones del "Consenso de Washington", en la convertibilidad de la moneda, la apertura externa y la reforma del Estado con miras a limitar el margen de maniobra de la política económica, fuente principal de inestabilidad según la visión de los responsables.

Inicialmente se estableció una banda de flotación —entre 8 y 10 mil australes- para luego establecer por Ley de Convertibilidad una paridad fija — de 10 mil australes por dólar-, la libre convertibilidad de la moneda, el respeto a la moneda fijada en los contratos, la anulación de las cláusulas indexatorias y la prohibición de emitir moneda sin respaldo. Pero la persistencia de la inflación por algún tiempo produjo un retraso cambiario que afectaría en lo sucesivo a la competitividad del sector exportador.

El plan tuvo un éxito inicial debido en parte al contexto internacional favorable dadas las bajas tasas internacionales y la mayor liquidez mundial; y mientras que la deuda externa disminuía temporalmente - merced al Plan Brady-, crecían la deuda interna y las deudas provinciales por la transferencia de servicios públicos sin la correspondiente partida presupuestaria. De manera tal que el nivel nacional de gobierno se desentendía de los costos sociales que sus políticas generaban, obligando a los gobiernos locales a hacerse cargo de los nuevos problemas, como el incremento de la pobreza y el desempleo (Passalacqua \& Villar, 1994). 


\section{Un modelo de cambio estructural con rigidez de precios}

El modelo de economía abierta con dos sectores - transable y no transablesupone que la transición de una situación de déficit comercial a otra con superávit requeriría de una caída en la absorción relativa del ingreso, de un desplazamiento de la producción de bienes no transables a transables y de una depreciación del tipo de cambio real, es decir, un aumento del precio relativo de los bienes transables (Sach $\&$ Larraín, 2002). Pero en una economía parcialmente industrializada, del tipo de "sustitución de importaciones" como la argentina, la combinación de liberalización comercial y apreciación del tipo de cambio trae consigo la aceleración del crecimiento en la productividad laboral de los sectores productores de bienes transables, como respuesta al shock externo, y al mismo tiempo un retraso en el crecimiento -o incluso una contracción - del empleo dada la mayor intensidad en la utilización de los restantes factores productivos (Frenkel \& González Rozada, 2000).

Ello obedece a que, bajo condiciones de rigidez de precios y salarios, el tipo de cambio real permanezca inalterado debido a que el tipo de cambio nominal permanece fijo y los precios de los bienes no transables presentan rigidez a la baja. El resultado de ello es que la caída en la producción de bienes no transables y en la absorción doméstica, no es compensada por un incremento en la producción de bienes transables, por lo cual la economía sufre un incremento del desempleo (Blanchard \& Pérez Enrri, 2000).
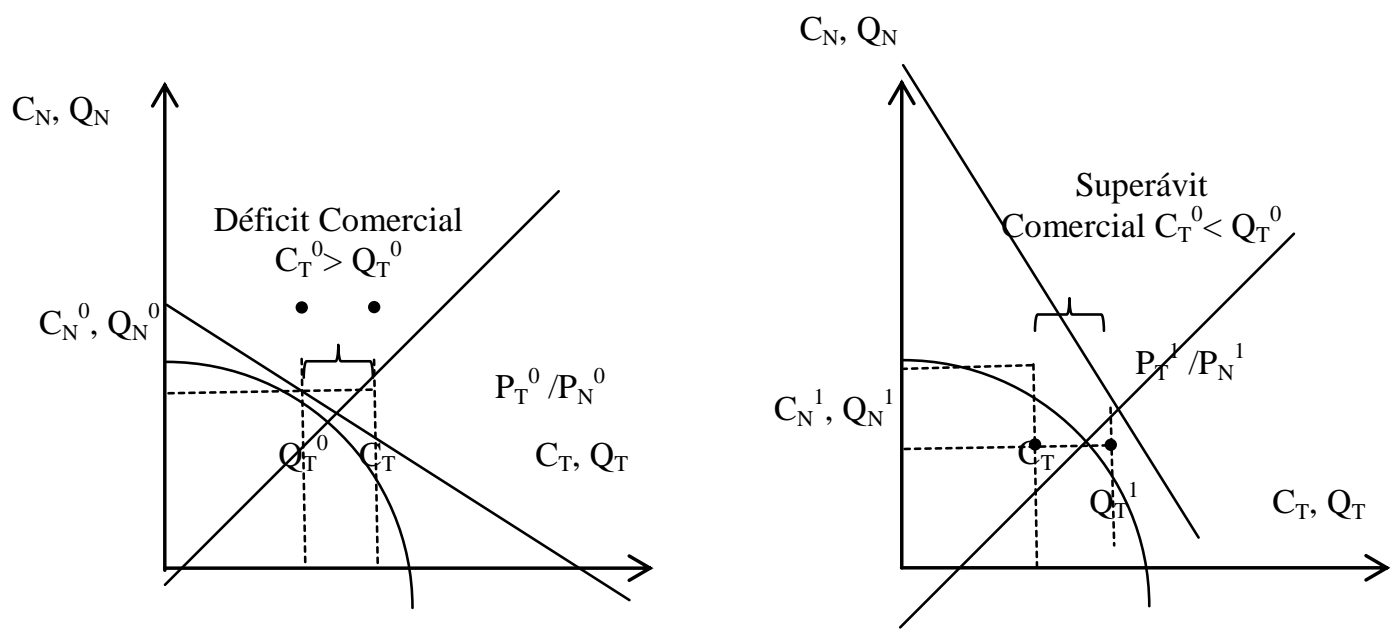

Otra consecuencia de la apertura comercial no contemplada por el modelo de dos sectores es el rol que juega la tecnología. De hecho, la apertura comercial genera un shock tecnológico que se traduce en un considerable grado de obsolescencia en buena parte del stock de capital, ya que el sector productivo que operaba con métodos atrasados con la protección de una economía cerrada debe enfrentar súbitamente a la 
competencia de productos ubicados en la frontera tecnológica. Ello incrementa los costos de la transición y se traduce en una caída en la demanda laboral en los sectores más afectados (Mazzitelli, 1997).

\section{Transformaciones estructurales en la Argentina}

Del análisis de los datos resulta que durante el período estudiado la producción de bienes transables se incrementó poco más de la mitad (6,34 \%) de lo que creció la economía en su conjunto (12,43\%) a raíz de la combinación de apertura comercial y sobrevaluación del tipo de cambio que tornaba poco competitivos los productos argentinos a nivel internacional. Dentro del sector productor de bienes se asistió a una primarización del aparato productivo debido a la fuerte expansión de actividades intensivas en recursos naturales y de escaso valor agregado (como el suministro de electricidad, gas y agua: 49,42 \% o la explotación de minas y canteras: 39,65 \%), en detrimento de la producción manufacturera (que se contrajo un 4,33 \%). Adicionalmente se produjo una tercerización de la economía debido a que las actividades de servicio se convirtieron en un refugio a la competencia externa, por lo que su desempeño superó (en más de 4 puntos porcentuales) al del total de la economía. Dicho crecimiento fue liderado por el sector de intermediación financiera $(59,16 \%)$, dado que, bajo el régimen de convertibilidad, tanto la cancelación de los pasivos externos como el mantenimiento de la cobertura total de la base monetaria requerían un creciente endeudamiento público a tasas de interés varias veces superiores a las internacionales, por lo que la Argentina se convirtió rápidamente en una atractiva plaza para las colocaciones financieras (Basualdo \& Kulfas, 2000). 
Cuadro 1: Evolución Sectorial del PIB. Variación acumulada 1993-2001

\begin{tabular}{|c|c|}
\hline \multirow[b]{2}{*}{ Agricultura, Ganadería y Silvicultura } & $26,49 \%$ \\
\hline & $22,85 \%$ \\
\hline Resto & $38,32 \%$ \\
\hline Pesca & $31,17 \%$ \\
\hline Explotación de Minas y Canteras & $39,65 \%$ \\
\hline Industria Manufacturera & $-4,33 \%$ \\
\hline Suministro de Electricidad, Gas y Agua & $49,42 \%$ \\
\hline Construcciones & $-1,47 \%$ \\
\hline SECTORES PRODUCTORES DE BIENES & $6,34 \%$ \\
\hline Comercio, Hoteles y Restaurantes & $4,35 \%$ \\
\hline Comercio Mayorista y Minorista & $1,02 \%$ \\
\hline Hoteles y Restaurantes & $23,24 \%$ \\
\hline \multicolumn{2}{|l|}{ Transporte, Comunicaciones e } \\
\hline Intermediación Financiera & $44,04 \%$ \\
\hline Transporte, Almacenamiento y & \\
\hline Comunicaciones & $34,85 \%$ \\
\hline Intermediación Financiera & $59,16 \%$ \\
\hline \multicolumn{2}{|l|}{ Actividad Inmobiliaria, Empresarial y de } \\
\hline Alquiler & $14,94 \%$ \\
\hline Administración Pública y Defensa & $-2,93 \%$ \\
\hline \multicolumn{2}{|l|}{ Enseñanza, Servicios Sociales y de } \\
\hline Salud & $21,50 \%$ \\
\hline Otras Actividades de Servicio & $19,68 \%$ \\
\hline \multicolumn{2}{|l|}{ SECTORES PRODUCTORES DE } \\
\hline SERVICIOS & $17,22 \%$ \\
\hline PRODUCTO INTERNO BRUTO & $12,43 \%$ \\
\hline
\end{tabular}

Fuente: elaboración propia con datos de la Dirección Nacional de Cuentas Nacionales. 
Cuadro 2: Participación del PBG de Corrientes en el PIB de Argentina

\begin{tabular}{|c|c|c|c|c|c|c|c|c|c|}
\hline & 1993 & 1994 & 1995 & 1996 & 1997 & 1998 & 1999 & $\begin{array}{l}20 \\
00\end{array}$ & $\begin{array}{l}20 \\
01\end{array}$ \\
\hline $\begin{array}{l}\text { Sector Primario } \\
\text { Agric., Ganad. y }\end{array}$ & $1,51 \%$ & $\begin{array}{r}1,77 \\
\% \\
2,26\end{array}$ & $1,92 \%$ & $1,93 \%$ & $1,80 \%$ & $\begin{array}{r}1,35 \\
\% \\
1,72\end{array}$ & $2,14 \%$ & $\begin{array}{r}1,9 \\
7 \% \\
2,5\end{array}$ & $\begin{array}{r}1,7 \\
9 \% \\
2,3\end{array}$ \\
\hline $\begin{array}{l}\text { Silvicultura } \\
\text { Resto }\end{array}$ & $0,36 \%$ & $\begin{array}{r}\% \\
0,36 \\
\%\end{array}$ & $2,50 \%$ & $2,58 \%$ & $2,38 \%$ & $\begin{array}{r}\% \\
0,34 \\
\%\end{array}$ & $2,71 \%$ & $\begin{array}{r}5 \% \\
0,3 \\
8 \%\end{array}$ & $\begin{array}{r}6 \% \\
0,3 \\
2 \%\end{array}$ \\
\hline Ind. Manufacturera & $1,30 \%$ & $\begin{array}{r}1,32 \\
\%\end{array}$ & $1,34 \%$ & $1,24 \%$ & $1,18 \%$ & $\begin{array}{r}1,18 \\
\%\end{array}$ & $1,23 \%$ & $\begin{array}{r}1,3 \\
1 \%\end{array}$ & $\begin{array}{r}1,2 \\
7 \%\end{array}$ \\
\hline Elec., Gas y Agua & $1,01 \%$ & $\begin{array}{r}0,94 \\
\%\end{array}$ & $1,77 \%$ & $1,65 \%$ & $1,81 \%$ & $\begin{array}{r}1,93 \\
\%\end{array}$ & $2,09 \%$ & $\begin{array}{r}2,0 \\
2 \%\end{array}$ & $\begin{array}{r}1,7 \\
2 \%\end{array}$ \\
\hline $\begin{array}{l}\text { Construcciones } \\
\text { SECTORES }\end{array}$ & $2,00 \%$ & $\begin{array}{r}1,84 \\
\%\end{array}$ & $2,92 \%$ & $1,85 \%$ & $1,72 \%$ & $\begin{array}{r}1,73 \\
\%\end{array}$ & $1,67 \%$ & $\begin{array}{r}1,5 \\
2 \%\end{array}$ & $\begin{array}{l}1,9 \\
3 \%\end{array}$ \\
\hline $\begin{array}{l}\text { PRODUCTORES DE } \\
\text { BIENES }\end{array}$ & $1,45 \%$ & $\begin{array}{r}1,48 \\
\% \\
\end{array}$ & $1,76 \%$ & $1,52 \%$ & $1,45 \%$ & $\begin{array}{r}1,37 \\
\% \\
\end{array}$ & $1,58 \%$ & $\begin{array}{r}1,5 \\
6 \%\end{array}$ & $\begin{array}{r}1,5 \\
4 \%\end{array}$ \\
\hline $\begin{array}{l}\text { Comercio, Hoteles y } \\
\text { Restaurantes } \\
\text { Transporte, } \\
\text { Comunicaciones e } \\
\text { Intermediación }\end{array}$ & $0,93 \%$ & $\begin{array}{r}1,01 \\
\%\end{array}$ & $1,05 \%$ & $1,04 \%$ & $1,03 \%$ & $\begin{array}{r}0,97 \\
\%\end{array}$ & $1,00 \%$ & $\begin{array}{r}0,9 \\
8 \%\end{array}$ & $\begin{array}{r}1,0 \\
0 \%\end{array}$ \\
\hline $\begin{array}{l}\text { Financiera } \\
\text { Actividades }\end{array}$ & $0,93 \%$ & $\%$ & $0,81 \%$ & $0,79 \%$ & $0,68 \%$ & $\%$ & $0,67 \%$ & $7 \%$ & $9 \%$ \\
\hline $\begin{array}{l}\text { Inmobiliarias y } \\
\text { empresariales }\end{array}$ & $1,57 \%$ & $\begin{array}{r}1,54 \\
\%\end{array}$ & $1,63 \%$ & $1,61 \%$ & $1,59 \%$ & $\begin{array}{r}1,59 \\
\%\end{array}$ & $1,61 \%$ & $\begin{array}{r}1,6 \\
0 \%\end{array}$ & $\begin{array}{r}1,6 \\
5 \%\end{array}$ \\
\hline $\begin{array}{l}\text { Administración } \\
\text { Pública, Defensa y }\end{array}$ & & 2,06 & & & & 2,13 & & 1,8 & 1,8 \\
\hline $\begin{array}{l}\text { Seguridad Social } \\
\text { Enseñanza, Servicios }\end{array}$ & $2,07 \%$ & $\begin{array}{r}\% \\
1,85\end{array}$ & $2,07 \%$ & $2,03 \%$ & $2,07 \%$ & $\begin{array}{r}\% \\
1,79\end{array}$ & $2,08 \%$ & $\begin{array}{r}5 \% \\
1,6\end{array}$ & $\begin{array}{r}5 \% \\
1,6\end{array}$ \\
\hline $\begin{array}{l}\text { Sociales y de Salud } \\
\text { Otras Actividades de }\end{array}$ & $1,81 \%$ & $\begin{array}{r}\% \\
0,78\end{array}$ & $1,70 \%$ & $1,71 \%$ & $1,75 \%$ & $\begin{array}{r}\% \\
0,97\end{array}$ & $1,79 \%$ & $\begin{array}{r}9 \% \\
1.0\end{array}$ & $\begin{array}{r}4 \% \\
10\end{array}$ \\
\hline Servicio & $0,82 \%$ & $\%$ & $0,92 \%$ & $0,93 \%$ & $0,89 \%$ & $\%$ & $1,03 \%$ & $1 \%$ & $1 \%$ \\
\hline SECTORES & $1,30 \%$ & 1,28 & $1,31 \%$ & $1,29 \%$ & $1,24 \%$ & 1,22 & $1,25 \%$ & 1,2 & 1,2 \\
\hline
\end{tabular}




\begin{tabular}{|c|c|c|c|c|c|c|c|c|c|}
\hline $\begin{array}{l}\text { PRODUCTORES DE } \\
\text { SERVICIOS }\end{array}$ & & $\%$ & & & & $\%$ & & $1 \%$ & $4 \%$ \\
\hline $\begin{array}{l}\text { PRODUCTO BRUTO } \\
\text { GEOGRÁFICO }\end{array}$ & $1,36 \%$ & $\begin{array}{r}1,36 \\
\%\end{array}$ & $1,48 \%$ & $1,38 \%$ & $1,33 \%$ & $\begin{array}{r}1,29 \\
\%\end{array}$ & $1,38 \%$ & $\begin{array}{r}1,3 \\
5 \%\end{array}$ & $\begin{array}{r}1,3 \\
6 \%\end{array}$ \\
\hline Población & $2,47 \%$ & $\begin{array}{r}2,47 \\
\%\end{array}$ & $2,47 \%$ & $2,47 \%$ & $2,48 \%$ & $\begin{array}{r}2,48 \\
\%\end{array}$ & $2,49 \%$ & $\begin{array}{r}2,4 \\
9 \%\end{array}$ & $\begin{array}{r}2,5 \\
1 \%\end{array}$ \\
\hline PBG per cápita & $55 \%$ & $55 \%$ & $60 \%$ & $56 \%$ & $54 \%$ & $52 \%$ & $56 \%$ & $\begin{array}{r}54 \\
\%\end{array}$ & $\begin{array}{r}54 \\
\%\end{array}$ \\
\hline
\end{tabular}

Fuente: elaboración propia con datos de la Dirección Nacional de Cuentas Nacionales y la Dirección de Estadísticas y Censos.

\section{Caracterización de la economía de Corrientes}

Una de las características más significativas de la economía de Corrientes durante los años estudiados viene dada por su escasa relevancia a nivel nacional. De los datos analizados surge que el producto bruto geográfico (PBG) de la provincia osciló entre el $1,29 \%$ y el $1,48 \%$ del producto interno bruto (PIB) del país. Si se considera a la superficie y a la población como indicadores aproximados de la dotación de recursos naturales y mano de obra con que cuenta la provincia, y teniendo en cuenta que Corrientes, con una superficie de $88.199 \mathrm{~km}^{2}$, representaba el 3,17 \% del territorio nacional y su población entre 1993 y 2001 pasó del 2,47 \% al 2,51 \% del total, resulta que la contribución de la economía correntina al producto nacional se situó sistemáticamente por debajo del aporte potencial dada su dotación de factores de la producción, lo cual explica que el PBG per cápita correntino oscilara entre el 52 \% y el $60 \%$ del PIB per cápita.

En cuanto a la composición sectorial de la economía correntina, corresponde hacer notar la importancia del sector primario en la producción de bienes, ya que, junto con la actividad manufacturera, comprende el $70,65 \%$ de la producción de bienes transable.

Cuadro 3: Estructura Sectorial Comparada PIB | PBG. Promedio 1993-2001

\begin{tabular}{|l|r|r|}
\hline & PIB & PBG \\
\hline Sector Primario & 7,60 & 10,03 \\
Agric., Ganad. y Silvicultura & $\%$ & $\%$ \\
Resto & 5,55 & 9,47 \\
& $\%$ & $\%$ \\
& 2,05 & 0,55
\end{tabular}




\begin{tabular}{|l|r|r|} 
& $\%$ & $\%$ \\
Ind. Manufacturera & 18,25 & 16,91 \\
Elec., Gas y Agua & $\%$ & $\%$ \\
& 2,49 & 3,09 \\
Construcciones & $\%$ & $\%$ \\
& 5,83 & 8,10 \\
SECTORES PRODUCTORES DE BIENES & $\%$ & $\%$ \\
& $\mathbf{3 4 , 1 6}$ & $\mathbf{3 8 , 1 3}$ \\
Comercio, Hoteles y Restaurantes & $\%$ & $\%$ \\
\hline Transporte, Comunicaciones e Intermediación & 17,41 & 12,79 \\
Financiera & 14,00 & $\%$ \\
Actividades Inmobiliarias y empresariales & $\%$ & $\%, 57$ \\
Administración Pública, Defensa y Seguridad & 15,56 & 18,23 \\
Social & $\%$ & $\%$ \\
Enseñanza, Servicios Sociales y de Salud & $\%, 81$ & 8,63 \\
Otras Actividades de Servicio & 8,30 & $\%$ \\
PBI | PBG & $\%$ & $\%$ \\
SECTORES PRODUCTORES DE SERVICIOS & 5,88 & 4,02 \\
\hline & $\mathbf{1 0 0 , 0}$ & $\mathbf{1 0 0 , 0}$ \\
\hline
\end{tabular}

Fuente: elaboración propia con datos de la Dirección Nacional de Cuentas Nacionales y la Dirección de Estadísticas y Censos.

Más específicamente, la producción ganadera se destaca dentro del sector primario como puede observarse al ver la preminencia que los pastizales tienen en el uso de las tierras productivas, con la cría de bovinos como actividad excluyente al concentrar el $76,66 \%$ del total de cabezas de ganado en la provincia. 
Cuadro 4: Distribución del uso de la tierra. Superficie de las EAP con límites definidos

\begin{tabular}{|l|r|r|}
\hline & \multicolumn{1}{|c|}{ Ctes. } & \multicolumn{1}{c|}{ Arg. } \\
\hline Superficie total: $6.850 .573 \mathrm{Ha}$ & $100,00 \%$ & $3,92 \%$ \\
Superficie implantada & & \\
$\quad$ Cultivos anuales & $1,13 \%$ & $0,40 \%$ \\
Cultivos perennes & $0,61 \%$ & $4,19 \%$ \\
Forrajeras anuales & $0,15 \%$ & $0,25 \%$ \\
Forrajeras perennes & $0,62 \%$ & $0,54 \%$ \\
Bosques y/o montes & $4,13 \%$ & $27,70 \%$ \\
Cultivos sin discriminar & $0,15 \%$ & $4,31 \%$ \\
Superficie destinada a otros usos & & \\
Pastizales & $78,42 \%$ & $5,85 \%$ \\
Bosques y/o montes naturales & $4,67 \%$ & $0,93 \%$ \\
Superficie apta no utilizada & $2,59 \%$ & $4,17 \%$ \\
Superficie no apta o de desperdicio & $7,04 \%$ & $5,75 \%$ \\
Caminos, parques y viviendas & $0,38 \%$ & $2,64 \%$ \\
Superficie sin discriminar & $0,10 \%$ & $0,53 \%$ \\
\hline
\end{tabular}

Fuente: elaboración propia con datos del Censo Nacional Agropecuario 2002

Cuadro 5: Distribución de EAP y cabezas de ganado por especie

\begin{tabular}{|l|r|r|}
\hline & \multicolumn{1}{|c|}{ Ctes. } & \multicolumn{1}{|c|}{ Arg. } \\
\hline EAP: 30.169 & $100,00 \%$ & $25,10 \%$ \\
Bovinos & $36,96 \%$ & $5,75 \%$ \\
Ovinos & $13,28 \%$ & $7,17 \%$ \\
Caprinos & $1,55 \%$ & $1,00 \%$ \\
Equinos & $39,48 \%$ & $6,95 \%$ \\
Procinos & $8,73 \%$ & $4,23 \%$ \\
Cabezas de ganado: 4.713 .579 & $100,00 \%$ & $28,07 \%$ \\
Bovinos & $76,66 \%$ & $7,44 \%$ \\
Ovinos & $18,66 \%$ & $7,00 \%$ \\
Caprinos & $0,20 \%$ & $0,23 \%$ \\
Equinos & $3,92 \%$ & $12,17 \%$ \\
Procinos & $0,56 \%$ & $1,22 \%$ \\
\hline
\end{tabular}

Fuente: elaboración propia con datos del Censo Nacional Agropecuario 2002 
En cuanto a las actividades de servicio cabe destacar la importancia del cuentapropismo como forma de inserción productiva dada la prevalencia del comercio y los servicios personales, que en conjunto abarcan el 65,87 \% de los locales relevados por el último Censo Nacional Económico disponible.

Cuadro 6: Locales por rama de actividad

\begin{tabular}{|c|c|c|}
\hline & Ctes. & $\begin{array}{l}\text { Arg } \\
\text {. }\end{array}$ \\
\hline \multirow{3}{*}{ Comercio al por mayor y menor, reparaciones } & 55,71 & 2,3 \\
\hline & $\%$ & $7 \%$ \\
\hline & 10,16 & 2,3 \\
\hline \multirow[t]{2}{*}{ Servicios comunitarios, Sociales y Personales } & $\%$ & $2 \%$ \\
\hline & 6,02 & 2,1 \\
\hline \multirow[t]{2}{*}{ Servicios sociales y de salud } & $\%$ & $7 \%$ \\
\hline & 5,50 & 1,9 \\
\hline \multirow[t]{2}{*}{ Servicios inmobiliarios, Empresariales y de Alquiler } & $\%$ & $3 \%$ \\
\hline & 4,96 & 1,5 \\
\hline Industria Manufacturera & $\%$ & $3 \%$ \\
\hline Servicio de Transporte, Almacenamiento y de & 3,93 & 1,9 \\
\hline \multirow[t]{2}{*}{ Comunicaciones } & $\%$ & $5 \%$ \\
\hline & 3,72 & 1,8 \\
\hline \multirow[t]{2}{*}{ Servicio de Hotelería y Restaurante } & $\%$ & $6 \%$ \\
\hline & 2,91 & 1,9 \\
\hline \multirow[t]{2}{*}{ Enseñanza } & $\%$ & $7 \%$ \\
\hline & 2,53 & 2,8 \\
\hline En proceso de clasificación & $\%$ & $3 \%$ \\
\hline Administración Pública, Defensa y Seguridad Social & 2,00 & 3,4 \\
\hline \multirow[t]{2}{*}{ Obligatoria } & $\%$ & $2 \%$ \\
\hline & 0,97 & 1,6 \\
\hline Unidades Auxiliares & $\%$ & $7 \%$ \\
\hline Intermediación financiera y Otros Servicios & 0,62 & 1,3 \\
\hline \multirow[t]{2}{*}{ Financieros } & $\%$ & $1 \%$ \\
\hline & 0,35 & 1,3 \\
\hline \multirow[t]{2}{*}{ Construcción } & $\%$ & $5 \%$ \\
\hline & 0,32 & 2,0 \\
\hline Agricultura, Ganadería, Caza y Silvicultura & $\%$ & $0 \%$ \\
\hline Electricidad, Gas y Agua & 0,29 & 3,6 \\
\hline
\end{tabular}




\begin{tabular}{|l|r|r|} 
& $\%$ & $3 \%$ \\
Explotación de minas y canteras & 0,01 & 0,6 \\
& $\%$ & $7 \%$ \\
Pesca y Servicios Conexos & 0,00 & 0,8 \\
\hline
\end{tabular}

Fuente: elaboración propia con datos del Censo Nacional Económico $2004 / 2005$

\section{Cambios en el entramado productivo provincial}

Al igual que lo apuntado precedentemente sobre los cambios en la composición morfológica de la economía nacional, la implementación del régimen de convertibilidad también trajo aparejadas profundas transformaciones en el entramado productivo provincial, aunque no siempre en el mismo sentido.

En primer lugar, puede señalarse el significativo crecimiento que experimentó la actividad ganadera durante el período (142,51 \%), lo cual es consistente con la información presentada previamente sobre uso del suelo y cantidad de cabezas de ganado.

En segundo término, cabe mencionar el crecimiento evidenciado en el rubro de suministro de electricidad, gas y agua, en el que las inversiones en el complejo hidroeléctrico de Yaciretá y la temprana privatización del servicio de agua potable, uno de los primeros en el sector junto con el de Buenos Aires, permitieron que la actividad creciera considerablemente (137,51 \%).

Capítulo aparte merece la importante contracción ocurrida en el sector estatal (13,41 \%) que, dada la transferencia a las provincias de los servicios educativos y sanitarios prestados anteriormente por el Estado nacional, puede verse como una forma de compensar presupuestariamente las nuevas competencias asumidas por la jurisdicción.

Cuadro 7: Evolución Sectorial del PBG. Variación

acumulada 1993-2001

\begin{tabular}{|c|r|}
\hline Sector Primario & $63,18 \%$ \\
Agricultura, Ganadería y Silvicultura & $65,37 \%$ \\
Agricultura & $60,66 \%$ \\
Ganadería & $142,84 \%$ \\
Silvicultura & $23,21 \%$ \\
Resto & $34,95 \%$ \\
Industria Manufacturera & $-7,31 \%$
\end{tabular}




\begin{tabular}{|l|r|} 
Electricidad, Gas y Agua & $137,51 \%$ \\
Construcciones & $\mathbf{7 , 0 3} \%$ \\
SECTORES PRODUCTORES DE BIENES & $\mathbf{1 4 , 1 4} \%$ \\
\hline Comercio, Hoteles y Restaurantes & $11,59 \%$ \\
Transporte, Comunicaciones e & \\
Intermediación Financiera & $12,57 \%$ \\
Actividades Inmobiliarias y empresariales & $19,50 \%$ \\
Administración Pública, Defensa y & \\
Seguridad Social & $-13,41 \%$ \\
Enseñanza, Servicios Sociales y de Salud & $12,19 \%$ \\
Enseñanza & $15,98 \%$ \\
Servicios Sociales y de salud & $7,85 \%$ \\
Otras Actividades de Servicio & $42,74 \%$ \\
Servicios Comunales, Sociales y & \\
Personales & $40,22 \%$ \\
Servicio Doméstico & $56,54 \%$ \\
SECTORES PRODUCTORES DE & \\
SERVICIOS & $\mathbf{1 1 , 9 8 \%}$ \\
\hline PRODUCTO BRUTO GEOGRÁFICO & $\mathbf{1 2 , 2 5 \%}$ \\
\hline
\end{tabular}

Fuente: elaboración propia con datos de la Dirección de Estadísticas y Censos

Al compararse los cambios operados en la economía correntina con las tendencias nacionales descriptas anteriormente surge que, aunque en ambos niveles la economía creció a tasas similares, en Corrientes la apertura comercial combinada con la apreciación del tipo de cambio no se tradujo en el trasvasamiento de la producción hacia actividades de servicio protegidas de la competencia externa, sino que, por el contrario, tanto la producción de bienes como de servicios crecieron de manera equilibrada. De hecho, el sector productor de bienes provincial creció más del doble $(14,14 \%)$ que su contraparte nacional (6,34 \%).

Lo que a primera vista parecería contradictorio resulta consistente con la primarización de la producción nacional, ya que al observar en detalle la evolución del sector puede demostrarse que los rubros que incidieron en el crecimiento del sector transable más significativamente fueron aquellos de escaso valor agregado, menos incididos por los costos internos y más dependientes de los precios fijados internacionalmente (v. g., ganadería, minas y canteras, etc.). En tanto que la actividad manufacturera, intensiva en mano de obra, sufrió una merma (7,31 \%) incluso mayor que la experimentada a nivel nacional (4,33\%). 
Cuadro 8: Evolución Sectorial Comparada PIB | PBG. Variación acumulada 1993-2001

\begin{tabular}{|c|c|c|}
\hline & PIB & PBG \\
\hline \multirow{2}{*}{ Sector Primario } & 26,49 & 63,18 \\
\hline & $\%$ & $\%$ \\
\hline \multirow{2}{*}{ Agricultura, Ganadería y Silvicultura } & 22,85 & 65,37 \\
\hline & $\%$ & $\%$ \\
\hline \multirow{2}{*}{ Resto } & 38,32 & 34,95 \\
\hline & $\%$ & $\%$ \\
\hline \multirow{2}{*}{ Industria Manufacturera } & $-4,33$ & $-7,31$ \\
\hline & $\%$ & $\%$ \\
\hline \multirow{2}{*}{ Electricidad, Gas y Agua } & 49,42 & 137,5 \\
\hline & $\%$ & $1 \%$ \\
\hline \multirow{2}{*}{ Construcciones } & $-1,47$ & 7,03 \\
\hline & $\%$ & $\%$ \\
\hline \multirow{2}{*}{ SECTORES PRODUCTORES DE BIENES } & 6,34 & 14,14 \\
\hline & $\%$ & $\%$ \\
\hline \multirow{2}{*}{ Comercio, Hoteles y Restaurantes } & 4,35 & 11,59 \\
\hline & $\%$ & $\%$ \\
\hline Transporte, Comunicaciones e & 44,04 & 12,57 \\
\hline Intermediación Financiera & $\%$ & $\%$ \\
\hline \multirow{2}{*}{ Actividades Inmobiliarias y empresariales } & 14,94 & 19,50 \\
\hline & $\%$ & $\%$ \\
\hline Administración Pública, Defensa y Seguridad & $-2,93$ & $-13,41$ \\
\hline Social & $\%$ & $\%$ \\
\hline \multirow{2}{*}{ Enseñanza, Servicios Sociales y de Salud } & 21,50 & 12,19 \\
\hline & $\%$ & $\%$ \\
\hline \multirow{3}{*}{ Otras Actividades de Servicio } & 19,68 & 42,74 \\
\hline & $\%$ & $\%$ \\
\hline & 17,22 & 11,98 \\
\hline \multirow[t]{2}{*}{ SECTORES PRODUCTORES DE SERVICIOS } & $\%$ & $\%$ \\
\hline & 12,43 & 12,25 \\
\hline PBI | PBG & $\%$ & $\%$ \\
\hline
\end{tabular}

Fuente: elaboración propia con datos de la Dirección Nacional de Cuentas Nacionales y la Dirección de Estadísticas y Censos 
En cuanto a las actividades de servicio, como se apuntó más arriba su desarrollo fue menor que a nivel nacional. Sin embargo, puede destacarse que, a diferencia de lo ocurrido a nivel nacional, Corrientes no compartió el auge financiero asociado a la moneda fuerte y el libre flujo de capitales, por lo cual el rubro de transporte, comunicaciones e intermediación financiera creció poco más de un tercio (12,57 \%) de lo que se incrementó a nivel nacional (44,04\%). Distinto fue el caso del rubro de comercio, hoteles y restaurantes, que mostró un dinamismo $(11,59 \%)$ más de dos veces superior a la media nacional (4,35\%), lo cual es consistente con una economía concentrada geográficamente y altamente dependiente de la actividad pública (Rofman \& García, 2007).

\section{Tipología del mercado laboral argentino}

Indudablemente uno de los aspectos más negativos del desempeño económico de la Argentina durante los años de vigencia del régimen de convertibilidad estuvo dado por el traumático comportamiento del mercado de trabajo, como puede observarse en la evolución que tuvieron los indicadores laborales pertinentes durante esos años (Boyer \& Neffa, 2004).

Uno de los fenómenos observados fue el inédito incremento del número de personas que se incorporaron a la fuerza de trabajo, por lo cual la tasa de actividad ascendió considerablemente. Entre las explicaciones esbozadas al respecto se encuentran aquellas que señalan una mayor propensión cultural a trabajar, que habría estado asociada a la incorporación de la mujer al mundo del trabajo, o aquellas que sostienen que los altos salarios habrían incrementado el costo de oportunidad para aquellos que permanecieran fuera del mercado de trabajo (Oliva, 2000). Una explicación alternativa, y más consistente con la información disponible, apunta al fenómeno conocido como "trabajador adicional", según el cual el aumento en la tasa de actividad habría estado relacionado con la disminución de los ingresos familiares en los estratos sociales de menores recursos, por lo que estos habrían respondido enviando un mayor número de sus integrantes a participar de la oferta de empleo (Torrado, 2004).

Los estudios realizados confirman que durante el período hubo un crecimiento proporcionalmente mayor de la tasa específica de actividad en los hogares de ingreso familiares per cápita más bajos. Puntualmente en los deciles inferiores la tasa específica de actividad creció 5,7 puntos porcentuales entre 1990 y 1998; mientras que para los deciles altos el crecimiento fue de 3,2 puntos porcentuales (Oliva, 2000).

Por su parte las transformaciones económicas a las que se hizo mención anteriormente implicaron que el entramado productivo nacional se volviera más intensivo en la utilización de capital y recursos naturales, y consecuentemente exhibiera 
una insuficiente capacidad de generación de puestos de trabajo, razón por la cual la tasa de empleo mostró un comportamiento negativo reduciéndose en dos puntos porcentuales a lo largo de esos años.

La combinación de una mayor presión en la oferta de fuerza laboral como estrategia de recomposición de ingresos por parte de las familias, conjuntamente con una demanda de trabajo menguante dada la insuficiente capacidad de generación de empleo por parte de las empresas, trajo como resultado un aumento inusitado del desempleo, que en 2001 superó el $17 \%$.

Cuadro 9: Evolución de Indicadores Laborales Argentina. Promedio onda Mayo y Octubre.

Total de aglomerados

\begin{tabular}{|l|r|c|c|c|c|c|c|c|c|}
\hline & $\mathbf{1 9 9 3}$ & $\mathbf{1 9 9 4}$ & $\mathbf{1 9 9 5}$ & $\mathbf{1 9 9 6}$ & $\mathbf{1 9 9 7}$ & $\mathbf{1 9 9 8}$ & $\mathbf{1 9 9 9}$ & $\mathbf{2 0 0 0}$ & $\mathbf{2 0 0 1}$ \\
\hline Tasa de & 41,25 & & & & & & & & \\
Actividad & $\%$ & $40,95 \%$ & $42,00 \%$ & $41,45 \%$ & $42,20 \%$ & $42,25 \%$ & $42,75 \%$ & $42,55 \%$ & $42,50 \%$ \\
$\begin{array}{l}\text { Tasa de } \\
\text { Empleo }\end{array}$ & $\begin{array}{r}\text { (19,25 } \\
\text { Tasa de } \\
\text { Desocupació }\end{array}$ & $36,25 \%$ & $34,65 \%$ & $34,30 \%$ & $35,90 \%$ & $36,90 \%$ & $36,70 \%$ & $36,20 \%$ & $35,15 \%$ \\
$\mathrm{n}$ & $9,60 \%$ & $11,45 \%$ & $17,50 \%$ & $17,20 \%$ & $14,90 \%$ & $12,80 \%$ & $14,15 \%$ & $15,05 \%$ & $17,35 \%$ \\
\hline
\end{tabular}

Fuente: elaboración propia con datos de la EPH. INDEC

\section{El mercado laboral correntino en perspectiva}

En cuanto al comportamiento del mercado laboral en Corrientes durante esos años, pueden señalarse similitudes y diferencias con las tendencias evidenciadas a nivel nacional. Por un lado existió el mismo incremento a nivel de la oferta de trabajo, y este tuvo una intensidad incluso superior en la provincia. En efecto, el crecimiento en el nivel de actividad (5,80 puntos porcentuales) fue cuatro veces y media superior al aumento nacional (1,25 puntos porcentuales), de manera tal que, aunque la tasa de actividad correntina se mantuvo sistemáticamente por debajo de su contraparte nacional, la brecha entre ambas se redujo entre 1993 y 2001. Las razones de por qué la tasa de actividad provincial fue comparativamente menor a la nacional podrían radicar en los menores salarios relativos pagados localmente, que habrían actuado como un estímulo, vía costo de oportunidad, de menor intensidad para la búsqueda de empleo; y de la misma manera el deterioro de las rentas familiares per cápita habría llevado a los sectores de menores recursos a buscar ingresos suplementarios en el mercado 
laboral con mayor intensidad, por lo que el crecimiento en la tasa de actividad fue mucho más pronunciado.

En cuanto a la tasa de empleo las tendencias fueron divergentes, mientras que a nivel nacional la cantidad de puestos de trabajo disminuyó (en 2,1 puntos porcentuales) como se apuntó más arriba, a nivel provincial hubo un ligero acrecentamiento de la tasa de empleo (1,05 puntos porcentuales). Nuevamente, si bien la tasa de empleo provincial se mantuvo sistemáticamente por debajo de su equivalente nacional, la distancia entre ambas alcanzó su mínimo en 1996 (4,2 puntos porcentuales) producto de las repercusiones que la crisis mexicana tuvo en la economía argentina.

Cuadro 10: Evolución de Indicadores Laborales Corrientes. Promedio onda mayo y octubre

\begin{tabular}{|l|r|r|r|r|r|r|r|r|r|}
\hline & $\mathbf{1 9 9}$ & $\mathbf{1 9 9}$ & $\mathbf{1 9 9}$ & $\mathbf{1 9 9}$ & $\mathbf{1 9 9}$ & $\mathbf{1 9 9}$ & $\mathbf{1 9 9}$ & $\mathbf{2 0 0}$ & $\mathbf{2}$ \\
& $\mathbf{3}$ & $\mathbf{4}$ & $\mathbf{5}$ & $\mathbf{6}$ & $\mathbf{7}$ & $\mathbf{8}$ & $\mathbf{9}$ & $\mathbf{0}$ & $\mathbf{2 0 0 1}$ \\
\hline \multirow{2}{*}{ Tasa de Actividad } & 31,1 & 31,8 & 35,7 & 35,1 & 36,1 & 36,1 & 36,0 & 35,1 & 36,95 \\
& $5 \%$ & $5 \%$ & $5 \%$ & $0 \%$ & $5 \%$ & $5 \%$ & $5 \%$ & $5 \%$ & $\%$ \\
Tasa de Empleo & 29,2 & 28,8 & 30,3 & 30,1 & 31,0 & 31,6 & 31,0 & 29,9 & 30,25 \\
& $0 \%$ & $0 \%$ & $5 \%$ & $0 \%$ & $5 \%$ & $5 \%$ & $0 \%$ & $0 \%$ & $\%$ \\
Tasa de & 6,20 & 9,60 & 15,1 & 14,1 & 14,1 & 12,6 & 13,9 & 15,0 & 18,20 \\
Desocupación & $\%$ & $\%$ & $0 \%$ & $5 \%$ & $5 \%$ & $0 \%$ & $0 \%$ & $5 \%$ & $\%$ \\
\hline
\end{tabular}

Fuente: elaboración propia con datos de la EPH. INDEC

Aunque el conjunto de empresas locales consiguió expandir la demanda laboral levemente mediante una reducida creación de puestos de trabajo, este crecimiento resultó insuficiente para hacer frente al importante aumento de la oferta de mano de obra, de manera que se asistió a un brutal incremento del desempleo a nivel provincial. La tasa de desocupación de Corrientes, que en el año 1993 representaba dos tercios de la tasa nacional (9,60 \%), llegó en el año 2000 a equiparar dicho guarismo (15,05 \%), para luego superarlo en el año 2001 (por 0,85 puntos porcentuales). De manera tal que en todo el período el crecimiento del desempleo provincial fue una vez y media superior al nacional.

Cuadro 11: Indicadores Laborales. Puntos porcentuales. Variación acumulada 1993-2001

\begin{tabular}{|l|c|c|}
\hline & Argentina & Corrientes \\
\hline Tasa de Actividad & 1,25 & 5,80 \\
Tasa de Empleo & $-2,10$ & 1,05 \\
Tasa de Desocupación & 7,75 & 12,00 \\
\hline
\end{tabular}

Fuente: elaboración propia con datos de la EPH. INDEC 


\section{Tendencias del empleo nacional}

De la información disponible sobre el número de asalariados registrados del sector privado desagregados por grandes divisiones puede obtenerse un esquema general de la dinámica interna del mercado laboral argentino durante el tramo final del régimen de convertibilidad de 1996 a 2001.

Luego de la turbulencia económica que significó la crisis mexicana de 1995, la producción de bienes decreció en el país un 0,89 \% hasta el año 2001. Sin embargo, en el mismo período la cantidad de asalariados en el sector se contrajo un 3,49 \% (más de 60 mil puestos de trabajo), indicando el perfil ahorrador de trabajo que adquirió el entramado productivo argentino.

En el mismo sentido, las cifras muestran cómo afectó la primarización del sector productor de bienes a la dinámica del empleo. Contrástese la disminución de $9 \%$ en el número de asalariados registrados en la producción manufacturera (una décima por encima de la propia contracción del producto sectorial), con el incremento de 17,36 \% en los puestos registrados en el sector de la pesca y de 9,40 \% en el sector de suministro de electricidad, gas y agua. Añádase que, incluso en estos últimos casos, la generación de empleo estuvo por debajo del crecimiento sectorial; especialmente para el suministro de electricidad, gas y agua fue de un tercio del crecimiento sectorial.

\section{Cuadro 12: Evolución sectorial del producto y del empleo en Argentina.}

PIB | Asalariados Registrados del sector privado. Variación acumulada 1996-2001

\begin{tabular}{|l|r|r|}
\hline & \multicolumn{1}{|c|}{ PIB } & \multicolumn{1}{|c|}{ Empleo } \\
\hline Sector Primario & $9,16 \%$ & $4,15 \%$ \\
Agricultura, Ganadería y Silvicultura & $10,89 \%$ & $4,01 \%$ \\
Resto & $5,93 \%$ & $5,64 \%$ \\
& & 17,36 \\
Pesca & $18,16 \%$ & $\%$ \\
$\quad$ Explotación de Minas y Canteras & $4,97 \%$ & $2,11 \%$ \\
Industria Manufacturera & $-8,11 \%$ & $-9,00 \%$ \\
Suministro de Electricidad, Gas y Agua & $27,10 \%$ & $9,40 \%$ \\
Construcciones & $-3,51 \%$ & $8,84 \%$ \\
SECTORES PRODUCTORES DE BIENES & & $-\mathbf{3 , 4 9}$ \\
& $-\mathbf{0 , 8 9} \%$ & $\%$ \\
\hline Comercio, Hoteles y Restaurantes & $-2,80 \%$ & 16,29
\end{tabular}




\begin{tabular}{|c|c|c|}
\hline Comercio Mayorista y Minorista & $-470 \%$ & $\begin{array}{r}\% \\
16,99 \\
\%\end{array}$ \\
\hline Hoteles y Restaurantes & $7,42 \%$ & $\begin{array}{r}12,71 \\
\%\end{array}$ \\
\hline Transporte, Comunicaciones e & & 19,26 \\
\hline Intermediación Financiera & $20,02 \%$ & $\%$ \\
\hline Transporte, Almacenamiento y & & 21,22 \\
\hline Comunicaciones & $15,93 \%$ & $\%$ \\
\hline Intermediación Financiera & $2638 \%$ & $\begin{array}{r}14,60 \\
\%\end{array}$ \\
\hline Actividad Inmobiliaria, Empresarial y de & & 29,41 \\
\hline Alquiler & $5,41 \%$ & $\%$ \\
\hline Enseñanza, Servicios Sociales y de Salud & $12,78 \%$ & $\begin{array}{r}32,52 \\
\%\end{array}$ \\
\hline Enseñanza & $s / d$ & $\begin{array}{r}63,32 \\
\%\end{array}$ \\
\hline Servicios Sociales y de Salud & $s / d$ & $0,11 \%$ \\
\hline Otras Actividades de Servicio & $13,30 \%$ & $\begin{array}{r}11,01 \\
\%\end{array}$ \\
\hline & & 21,54 \\
\hline SECTORES PRODUCTORES DE SERVICIOS & $7,32 \%$ & $\%$ \\
\hline $\begin{array}{l}\text { PIB | Total Asalariados Registrados del } \\
\text { sector privado }\end{array}$ & $3,90 \%$ & $\begin{array}{r}11,58 \\
\%\end{array}$ \\
\hline
\end{tabular}

Fuente: elaboración propia a partir de datos de la Dirección Nacional de Cuentas Nacionales (INDEC) y del Observatorio de Empleo y Dinámica Empresarial (MTEySS)

El área de servicio mostró un comportamiento totalmente opuesto, ya que allí se produjo un incremento en la cantidad de asalariados registrados $(21,54 \%)$ que incluso casi triplicó al propio crecimiento del producto (7,32 \%) explicitando cómo el sector de producción no transable se convirtió en un refugio contra la competencia internacional tanto para empresarios como para trabajadores.

La mayor generación de asalariados registrados se dio en el sector de enseñanza con un $63 \%$ (equivalente a más de 120 mil puestos de trabajo), seguido por la actividad inmobiliaria, empresarial y de alquiler con un $29,41 \%$ de suba (más que quintuplicando el crecimiento del producto sectorial). 
Así como los cambios operados en el sector productor de bienes lo volvieron menos propenso a la utilización de mano de obra, en los servicios se dio un uso intensivo de dicho factor productivo de manera tal que en la mayoría de los rubros la cantidad de puestos de trabajo generados superó a las tasas de crecimiento del producto sectorial específico. La excepción más notable estuvo dada por la intermediación financiera, gracias a la cual, a pesar de las sucesivas crisis internacionales ocurridas a partir de 1995, ese sector se las ingenió para seguir creciendo un 26,38 \%, y sin embargo, el empleo en el área aumentó poco más de la mitad de dicha cifra (14,60 \%).

\section{Tendencias del empleo provincial}

En cuanto la dinámica del empleo en Corrientes entre los años 1996 y 2001 puede observarse que la inserción productiva que ensayó la provincia le permitió sortear la fase recesiva con un ligero aumento de la producción de bienes $(0,5 \%)$, pero a costa de una fuerte contracción en el número de asalariados (4,24 \%).

A nivel provincial la primarización en la producción de bienes fue aun más patente, pues mientras el empleo manufacturero se contrajo ligeramente $(0,46 \%)$, en el sector de pesca prácticamente se cuadruplicó (388,89\%), seguido por la explotación de minas y canteras $(65,05 \%)$ donde la actividad excluyente vino dada por la extracción de arena y basalto para la construcción (DEyC, 2009). Especial mención debe hacerse del sector de la construcción, tradicional empleador de mano de obra, que durante el período redujo su personal considerablemente (21,55 \%) a pesar del leve crecimiento sectorial (1,56\%). 


\section{Cuadro: Evolución Sectorial del Producto y del Empleo en}

\section{Corrientes.}

PBG | Asalariados Registrados del sector privado. Variación acumulada 1996-2001

\begin{tabular}{|c|c|c|}
\hline & PBG & $\begin{array}{c}\text { Emple } \\
\text { o }\end{array}$ \\
\hline \multirow{2}{*}{ Sector Primario } & 17,71 & \\
\hline & $\%$ & $-0,73 \%$ \\
\hline \multirow{2}{*}{ Agricultura, Ganadería y Silvicultura } & 18,84 & \\
\hline & $\%$ & $-1,36 \%$ \\
\hline \multirow{2}{*}{ Resto } & 5,77 & 65,11 \\
\hline & $\%$ & $\%$ \\
\hline \multirow{2}{*}{ Pesca } & & 388,89 \\
\hline & $s / d$ & $\%$ \\
\hline \multirow{2}{*}{ Explotación de Minas y Canteras } & & 65,05 \\
\hline & $s / d$ & $\%$ \\
\hline \multirow{2}{*}{ Industria Manufacturera } & $-6,17$ & \\
\hline & $\%$ & $-0,46 \%$ \\
\hline \multirow{2}{*}{ Suministro de Electricidad, Gas y Agua } & 34,77 & 19,70 \\
\hline & $\%$ & $\%$ \\
\hline \multirow{2}{*}{ Construcciones } & 1,56 & $-21,55$ \\
\hline & $\%$ & $\%$ \\
\hline \multirow{2}{*}{ SECTORES PRODUCTORES DE BIENES } & 0,50 & $-4,24$ \\
\hline & $\%$ & $\%$ \\
\hline \multirow{2}{*}{ Comercio, Hoteles y Restaurantes } & $-7,31$ & 13,30 \\
\hline & $\%$ & $\%$ \\
\hline \multirow{2}{*}{ Comercio Mayorista y Minorista } & & 15,07 \\
\hline & $s / d$ & $\%$ \\
\hline Hoteles y Restaurantes & $s / d$ & $-1,76 \%$ \\
\hline Transporte, Comunicaciones e & 5,46 & 26,48 \\
\hline Intermediación Financiera & $\%$ & $\%$ \\
\hline Transporte, Almacenamiento y & & 12,83 \\
\hline Comunicaciones & $s / d$ & $\%$ \\
\hline \multirow{2}{*}{ Intermediación Financiera } & & 100,16 \\
\hline & $s / d$ & $\%$ \\
\hline Actividad Inmobiliaria, Empresarial y de & 7,84 & 24,50 \\
\hline Alquiler & $\%$ & $\%$ \\
\hline Enseñanza, Servicios Sociales y de Salud & 8,83 & 25,04 \\
\hline
\end{tabular}




\begin{tabular}{|l|r|r|} 
& $\%$ & $\%$ \\
Enseñanza & 18,38 & 35,34 \\
Servicios Sociales y de Salud & $\%$ & $\%$ \\
& $-3,13$ & 13,07 \\
Otras Actividades de Servicio & $\%$ & $\%$ \\
& 22,31 & \\
SECTORES PRODUCTORES DE SERVICIOS & $\%$ & $5,13 \%$ \\
\hline PBG I Total Asalariados Registrados del & $\mathbf{1 , 9 9}$ & $\mathbf{1 7 , 8 8}$ \\
sector privado & \% & $\mathbf{6 , 8 6} \%$ \\
\hline
\end{tabular}

Fuente: elaboración propia a partir de datos de la Dirección de Estadísticas y Censos y del Observatorio de Empleo y Dinámica Empresarial (MTEySS)

Por su parte los servicios tuvieron un comportamiento que, aunque no tan significativo como el nacional, tuvo los mismos rasgos generales. El crecimiento global del empleo en los servicios (17,88 \%) más que cuadruplicó el incremento del producto sectorial (3,09 \%). Dentro del sector el rubro más destacado estuvo dado por la intermediación financiera, que duplicó su plantel (100,16 \%), seguida por el rubro de enseñanza (35,34\%).

\section{A modo de conclusiones}

Aunque el neoliberalismo de finales del siglo XX planteaba la no intervención estatal como condición de posibilidad para desencadenar las fuerzas productivas de la economía en orden a promover el desarrollo de las naciones, sería un error asociar esta defensa del Estado ausente (o mínimo) con tentativas de instalar una especie de "estado neutral", entendido este como algún tipo de arreglo económico-institucional basado exclusivamente en las fuerzas del mercado.

En rigor de verdad, la puesta en práctica de las reformas estructurales en la Argentina a comienzos de la década de 1990 estuvo lejos de ser neutral. Por el contrario, las medidas adoptadas tuvieron como consecuencia la destrucción de una sustancial parte del tejido industrial (primarización de la producción de bienes), así como el sobredimensionamiento de la actividad de intermediación financiera (financiarización de los servicios). Efectos estos consistentes con el modelo propuesto de economía abierta con dos sectores. 
Afrontar el estudio de las transformaciones económicas operadas durante la vigencia del régimen de convertibilidad en la Argentina y sus efectos sobre el mercado laboral desde una perspectiva a la vez regional y sectorial reporta una serie de ventajas respecto de los abordajes tradicionales en la materia. En primer lugar, puede indicarse que ello permite reconocer la situación diferencial en que se encuentran los actores que componen los diversos entramados productivos existentes, examinar las relaciones asimétricas de negociación entre estos y discernir los factores explicativos asociados a las propias estructuras de aquellos relacionados con su particular forma de inserción.

En ese sentido pueden apuntarse los casos de la industria manufacturera y los servicios financieros, ejemplos paradigmáticos aunque por razones opuestas, el primero de ellos porque a partir de 1996 logró conservar las fuentes laborales, en un contexto nacional de destrucción de puestos de trabajo, a costa de reducciones salariales, y el segundo porque en las mismas circunstancias fue capaz de mantener el nivel de las remuneraciones a nivel provincial, a pesar de los drásticos recortes nacionales.

Otra de las ventajas del abordaje analítico aquí presentado radica en que permite conservar una visión global sobre la situación de conjunto. Así pudo señalarse cómo las transformaciones experimentadas a nivel local, aunque divergentes en ciertos aspectos de los procesos nacionales, respondían al mismo intento de reconfigurar la matriz productiva sobre la base de un patrón de desarrollo orientado hacia el exterior, de manera tal que la economía provincial devino en una imagen especular de la economía argentina en la convertibilidad.

Sintetizando, el patrón de especialización ensayado por la provincia de Corrientes durante esos años estuvo en sintonía con los lineamientos del programa económico implementado (crecimiento basado en exportaciones primarias) y tuvo éxito en aprovechar las "ventajas" que ofreció a partir de su puesta en marcha, de manera que esos primeros años fueron testigos de un fuerte crecimiento económico. Así mismo, la provincia sufrió con mayor intensidad los efectos del agotamiento del modelo a partir de 1996.

Quedará para una instancia posterior completar el análisis incluyendo los efectos que el régimen de convertibilidad tuvo sobre el empleo informal a nivel local y los intentos de morigerar las consecuencias sociales probados por el Estado provincial, aspectos ineludibles a la hora de brindar una visión acabada del período. 


\section{Bibliografía}

Barbero, M. I., Saborido, J., Beremblum, R., López Nadal, G., \& Ojeda, G. (2007). Historia Económica Mundial. Del paleolítico a Internet. Buenos Aires: Emecé.

Basualdo, E., \& Kulfas, M. (Julio-Agosto de 2000). Fuga de capitales y endeudamiento externo en la Argentina. Realidad Económica(173), 76-103.

Blanchard, O., \& Pérez Enrri, D. (2000). Macroeconomía. Teoría y política económica con aplicaciones a América Latina. Buenos Aires: Prentice-Hall.

Boyer, R., \& Neffa, J. (2004). La economía argentina y sus crisis (1976-2001): Visiones institucionalistas y regulacionistas. Buenos Aires: Miño y Dávila.

DEyC. (2009). Producto Bruto Geográfico. Dirección de Estadísticas y Censos / CEPAL. Período 1993 / 2008. Corrientes: DEyC.

Ferrer, A. (2006). Hechos y ficciones de la globalización: Argentina y el Mercosur en el sistema internacional (Quinta ed.). Buenos Aires: Fondo de Cultura Económica.

Frenkel, R., \& González Rozada, M. (2000). Liberalización del balance de pagos. Efectos sobre el crecimiento, el empleo y los ingresos en Argentina. Buenos Aires: CEDES.

Krugman, P., \& Obstfeld, M. (2002). Economía internacional: Teoría y política del comercio internacional. Buenos Aires: Emecé.

Mazzitelli, S. (1997). Desempleo Regional. Una consideración de un modelo de dos sectores. Buenos Aires: Insituto Superior de Economistas de Gobierno.

Oliva, M. (2000). Consecuencias de las políticas públicas sobre el mercado laboral en Argentina en el período 1989 - 1999. En J. Lindenboim, Cuadernos del CEPED N 4: Crisis y metamorfosis del mercado de trabajo (págs. 43-56). Buenos Aires: CEPED.

Ossona, J. (2001). Empresarios, Estado y estabilidad (1989-1993): De la hiperinflación a la estabilidad neoliberal. Buenos Aires: Ediciones del Centro de Estudiantes de la Facultad de Ciencias Económicas, Universidad de Buenos Aires.

Passalacqua, E., \& Villar, A. (Noviembre de 1994). La política social de los municipios argentinos : Un panorama global. Revista de Ciencias Sociales(1), 147-188.

Rapoport, M. (2005). Historia económica, política y social de la Argentina (18802003). Buenos Aires: Ariel.

Rofman, A., \& García, A. (2007). Las economías regionales después de la convertibilidad: entre cambios y continuidades. Los casos del tabaco burley (Misiones), la fruticultura (Río Negro), el algodón (Chaco) y la vitivinicultura (San Juan y Mendoza). En J. Neffa, \& R. Boyer, Escenarios de salida de crisis y estrategias alternativas de desarrollo para Argentina (págs. 545-569). Buenos Aires: Miño y Dávila.

Sach, J., \& Larraín, F. (2002). Macroeconomía en la economía global (Segunda ed.). Buenos Aires: Pearson Education.

Thorp, R. (1998). Progreso, pobreza y exclusión: Una historia económica de América Latina en el siglo XX. Washington: BID. 
De Prácticas y discursos/ Universidad Nacional del Nordeste/ Centro de Estudios Sociales

Torrado, S. (2004). La herencia del ajuste. Cambios en la sociedad y en la familia. Buenos Aires: Capital Intelectual.

Vitelli, G. (1999). Los dos siglos de la Argentina: Historia económica comparada. Buenos Aires: Prendergast. 